FACTA UNIVERSITATIS

Series: Mechanical Engineering Vol. 16, No 2, 2018, pp. 99 - 113

https://doi.org/10.22190/FUME180327013P

Original scientific paper

\title{
METHOD OF DIMENSIONALITY REDUCTION IN CONTACT MECHANICS AND FRICTION: A USER'S HANDBOOK. III. VISCOELASTIC CONTACTS
}

\author{
UDC 539.3
}

\author{
Valentin L. Popov ${ }^{1,2,3}$, Emanuel Willert ${ }^{1}$, Markus Heß ${ }^{1}$ \\ ${ }^{1}$ Technische Universität Berlin, Berlin, Germany \\ ${ }^{2}$ National Research Tomsk Polytechnic University, Tomsk, Russia \\ ${ }^{3}$ National Research Tomsk State University, Tomsk, Russia
}

\begin{abstract}
Until recently the analysis of contacts in tribological systems usually required the solution of complicated boundary value problems of three-dimensional elasticity and was thus mathematically and numerically costly. With the development of the so-called Method of Dimensionality Reduction (MDR) large groups of contact problems have been, by sets of specific rules, exactly led back to the elementary systems whose study requires only simple algebraic operations and elementary calculus. The mapping rules for axisymmetric contact problems of elastic bodies have been presented and illustrated in the previously published parts of The User's Manual, I and II, in Facta Universitatis series Mechanical Engineering [5, 9]. The present paper is dedicated to axisymmetric contacts of viscoelastic materials. All the mapping rules of the method are given and illustrated by examples.
\end{abstract}

Key Words: Contact, Friction, Viscoelasticity, Rheology, Method of Dimensionality Reduction

\section{INTRODUCTION}

In recent years the Method of Dimensionality Reduction (MDR) has been developed to efficiently deal with axisymmetric [1] and non-axisymmetric contacts [2]. The scope of applicability includes normal contacts with and without adhesion as well as tangential contacts [1], torsional contacts [3], contacts of Functionally Graded Materials [4-5] and viscoelastic contacts [6-8]. In preceding papers the mapping rules of MDR have been

Received March 27, 2018 / Accepted May 11, 2018

Corresponding author: Emanuel Willert

Technische Universität Berlin, Sekr. C8-4, Straße des 17. Juni 135, D-10623 Berlin

E-mail: e.willert@tu-berlin.de 
summarised and illustrated for axisymmetric contacts with a compact contact area for homogeneous [9] and power-law graded [5] elastic materials. The present publication gives a similar "user's manual" for the treatment of viscoelastic contacts.

\section{BASIC ASSUMPTIONS}

Let us in the beginning briefly clarify the fundamental assumptions, which define the framework of our method. The results of the set of simple rules given in this paper to solve axisymmetric contact problems of viscoelastic materials will be exactly correct, if all assumptions are met. Yet the method can also be used if some of the assumptions are broken although in this case the obtained solutions might exhibit smaller or larger errors, depending on the precise circumstances.

Firstly, we only consider homogeneous, isotropic, linear-viscoelastic media. Moreover, the deformations have to be small to ensure kinematic linearity. In this case we are also allowed to work within the half-space approximation. Throughout most of the paper we will additionally demand incompressible material behaviour, i.e. Poisson ratio $v$ shall be equal to 0.5. The treatment of compressible materials will, as far as possible, be covered in a separate section.

Under these assumptions the viscoelastic material can be described by a single timedependent shear relaxation function $G(t)$, which gives the material's stress response to a unit strain increment. Stress response $\sigma(t)$ to an arbitrary deformation history $\gamma(t)$ is due to the superposition principle given by the sum of stresses for all past strain increments ([10], p.257),

$$
\sigma(t)=\int G\left(t-t^{\prime}\right) \mathrm{d} \gamma=\int_{-\infty}^{t} G\left(t-t^{\prime}\right) \dot{\gamma}\left(t^{\prime}\right) \mathrm{d} t^{\prime} .
$$

As the convolution (1) in the time domain corresponds to a product in the Laplace domain, Eq. (1) can alternatively be written in the following way:

$$
\sigma^{*}(s)=G^{*}(s) s \gamma^{*}(s)
$$

whereas a star denotes the Laplace transform into the $s$-domain, i.e.

$$
G^{*}(s):=\int_{0}^{\infty} G(t) \exp (-s t) \mathrm{d} s
$$

and similarly for all others.

Also, a time-dependent shear creep function $J(t)$, i.e. the strain response to a unit stress increment, can be defined ([11], p.215). The strain response to an arbitrary stress history is, analogously to Eq. (1), given by

$$
\gamma(t)=\int_{-\infty}^{t} J\left(t-t^{\prime}\right) \dot{\sigma}\left(t^{\prime}\right) \mathrm{d} t^{\prime}
$$

or, equivalently, in the Laplace domain 


$$
\gamma^{*}(s)=J^{*}(s) s \sigma^{*}(s)
$$

Hence both the material functions are coupled by the relation

$$
s^{2} G^{*}(s) J^{*}(s) \equiv 1 .
$$

In case of harmonic oscillations, or put generally, in the frequency domain, for any linear viscoelastic material there is a linear proportionality between (shear) stress and (shear) strain - this directly follows from the material law in Eq. (1). The coefficient of proportionality is called "complex dynamic modulus"

$$
\hat{G}(\omega):=i \omega \int_{0}^{\infty} G(t) \exp (-i \omega t) \mathrm{d} t=i \omega G^{*}(s=i \omega) .
$$

Its real part is referred to as "storage modulus" and the imaginary part as "loss modulus".

For the contact of two viscoelastic bodies with creep functions $J_{1}$ and $J_{2}$ both the creep functions simply have to be linearly superposed,

$$
J(t)=J_{1}(t)+J_{2}(t) .
$$

Then a combined shear relaxation function $G(t)$ can also be defined via Eq. (6).

Finally, we will only consider quasi-static processes, i.e. all characteristic velocities of the contact problem must be much smaller than the smallest speed of wave propagation in the viscoelastic medium, and neglect adhesion or plasticity.

\section{RHEOLOGICAL MODELS}

The viscoelastic properties of materials, as they have been briefly introduced in the previous section, are often represented in terms of rheological models. The basic elements of those models are a spring, representing ideally elastic properties, and a dashpot, representing ideally viscous properties.

Combining a sufficiently large set of those basic elements, any arbitrarily complex (linear-) viscoelastic behaviour can be captured. Thereby only two fundamental rules of superposition exist: for two elements in parallel to each other, the respective relaxation functions have to be linearly superposed; for two elements in series the creep functions are superposed. The rheological model, which reproduces a given relaxation function $G(t)$ shall henceforth in this paper be denoted with a simple box accompanied by the respective relaxation function (see Tab. 1).

We should point out that all the values of stiffness and damping in these models are to be understood as continuum variables, i.e. per unit volume, which is why we will always speak of moduli and viscosities.

Tab. 1 shows a compilation of the most commonly used viscoelastic material models and their rheological representations as well as the associated relaxation and creep functions. Thereby $\delta(t)$ denotes the Dirac $\delta$-distribution while all the other designations are self-explanatory, based on the depicted rheological models. 
Table 1 Rheological representations and material functions for the most common linear viscoelastic material models ( $\delta$ denotes the Dirac $\delta$-distribution)

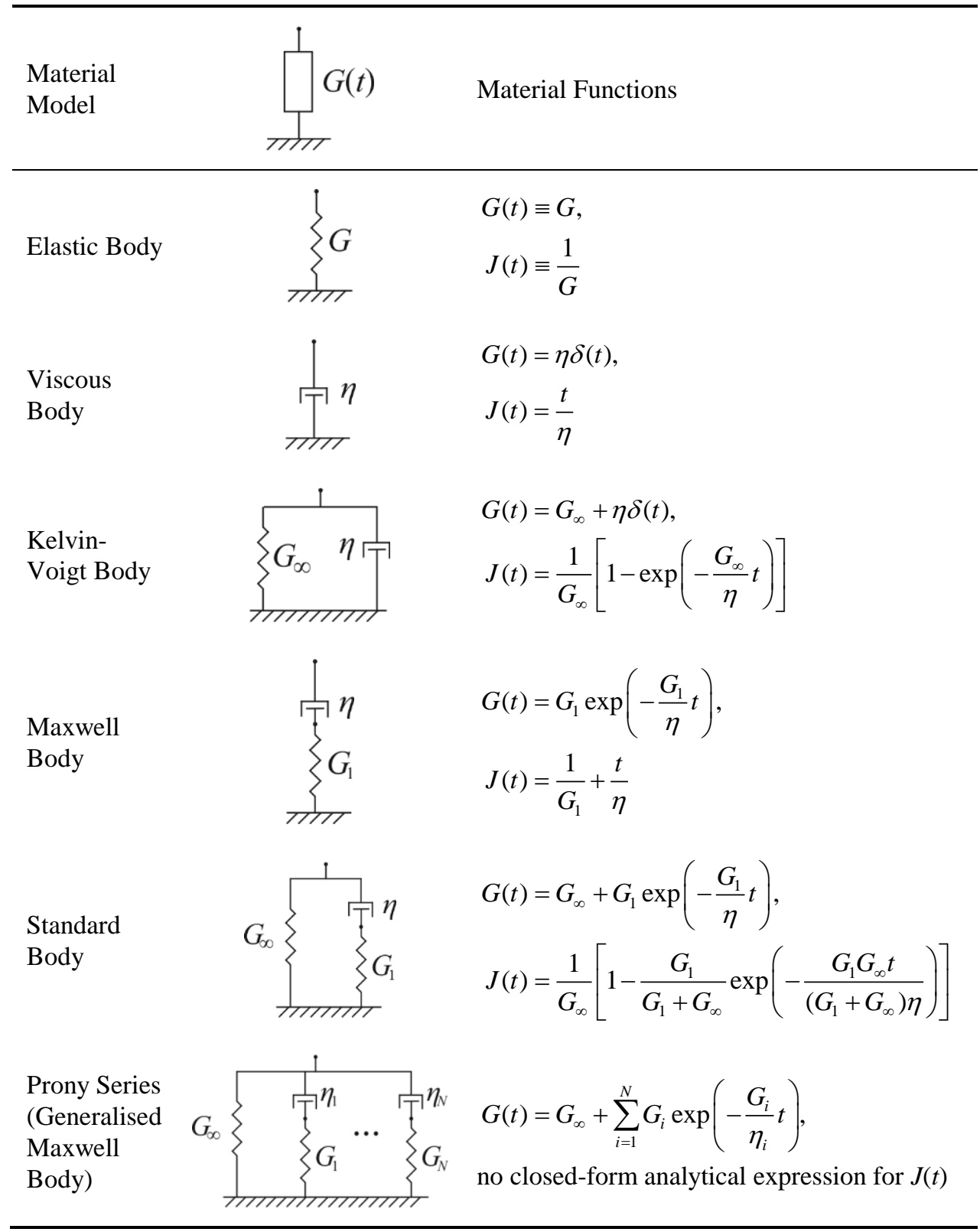




\section{TWO PREPARATORY STEPS OF THE METHOD}

We consider the contact of two incompressible viscoelastic bodies with combined shear relaxation function $G(t)$ (or, equivalently, combined creep function $J$ ). The nondeformed gap between both the bodies shall be an axisymmetric function $z=f(r)$. To solve contact problems of this configuration within the MDR two introductory steps are necessary.

Firstly, the axisymmetric gap has to be transformed into an equivalent (rigid) plain profile $g(x)$ by the integral transform [9]

$$
g(x)=|x| \int_{0}^{|x|} \frac{f^{\prime}(r) \mathrm{d} r}{\sqrt{x^{2}-r^{2}}} .
$$

This relation is the same as in the elastic case and is thus explained and illustrated by several examples in the first part of this user's manual. The inverse transform of Eq. (9) reads [9]

$$
f(r)=\frac{2}{\pi} \int_{0}^{r} \frac{g(x) \mathrm{d} x}{\sqrt{r^{2}-x^{2}}} .
$$

Secondly, a one-dimensional foundation of independent, linear-viscoelastic elements, each in distance $\Delta x$ of each other, must be initialised, as demonstrated in Fig. 1.

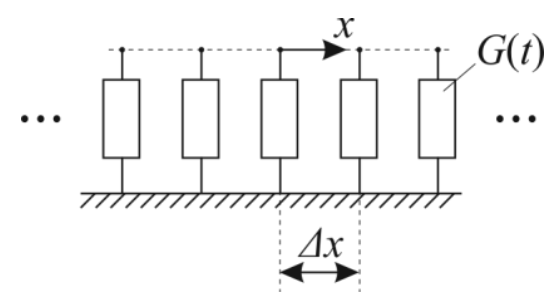

Fig. 1 One-dimensional foundation of linear-viscoelastic elements

A single element of the foundation is given by the rheological model for relaxation function $G(t)$, as shown in the previous section. For example, if the relaxation behaviour can be captured by a three-element standard solid, a single element of the viscoelastic foundation is given by a spring in series with a dashpot, the pair in parallel with another spring, as described above. The elements will have time-dependent values of normal and tangential stiffness (note that we assume incompressibility, i.e. $v=0.5$ ),

$$
\begin{aligned}
& \Delta k_{z}(t)=\frac{2}{1-v} G(t) \Delta x=4 G(t) \Delta x, \\
& \Delta k_{x}(t)=\frac{4}{2-v} G(t) \Delta x=\frac{8}{3} G(t) \Delta x,
\end{aligned}
$$

or in the frequency domain, 


$$
\begin{aligned}
\Delta \hat{k}_{z}(\omega) & =4 \hat{G}(\omega) \Delta x, \\
\Delta \hat{k}_{x}(\omega) & =\frac{8}{3} \hat{G}(\omega) \Delta x .
\end{aligned}
$$

\section{NORMAL CONTACT OF AXISYMMETRIC BODIES}

The equivalent profile defined by Eq. (9) is now pressed into the viscoelastic foundation defined by Eq. (11) by an indentation depth $d(t)$. Without loss of generality we will assume that the indentation starts at time $t=0$, and that the viscoelastic medium previously was stress-free and non-deformed. Vertical displacement $w_{1 \mathrm{D}}$ of an element at position $x$ within the contact area of radius $a$ is enforced by the indentation,

$$
w_{1 \mathrm{D}}(x, t)=d(t)-g(x), \quad|x| \leq a .
$$

An element comes into contact (geometrically) if the displacement of the non-contacting surface equals the displacement enforced by the indentation, i.e.

$$
w_{1 \mathrm{D}}^{\text {n.c. }}(a(t), t)=d(t)-g(a(t)) \text {. }
$$

For a monotonically increasing contact radius the non-contacting surface is not deformed. In this case the contact radius is therefore simply given by the relation

$$
d(t)=g(a(t)),
$$

which, for a monotonically increasing contact radius, is a universal relation independent of the material rheology, as proven by Lee \& Radok [12]. If the contact radius has extremal values the creep behaviour of the area without direct contact must be traced and inserted into Eq. (14) to give correct results [6].

The normal force in a single element of the foundation is due to the superposition principle given by

$$
\Delta F_{N}(x, t)=4 \Delta x \int_{0}^{t} G\left(t-t^{\prime}\right) \frac{\partial w_{1 \mathrm{D}}\left(x, t^{\prime}\right)}{\partial t^{\prime}} \mathrm{d} t^{\prime},
$$

or in the frequency domain

$$
\Delta \hat{F}_{N}(x, \omega)=4 \Delta x \hat{G}(\omega) \hat{w}_{1 \mathrm{D}}(x, \omega) .
$$

Note that if the rheological model contains separate dashpots, like the Kelvin-Voigt model, stress relaxation function $G(t)$ includes Dirac-distributions, which have to be evaluated in the integral using their filter properties. Instead of evaluating Eq. (16), which under some circumstances may require the knowledge about the entire loading history, one can also apply the complete set of equilibrium conditions for the single element, including the inner degrees of freedom. For example, the standard element shown on the left of Fig. 2 has one inner degree of freedom representing the material relaxation. The equilibrium conditions for the outer and inner degree of freedom are 


$$
\begin{aligned}
\Delta F_{N} & =4 \Delta x\left(G_{\infty} w_{1 \mathrm{D}}+G_{1} \tilde{w}_{1 \mathrm{D}}\right), \\
0 & =\eta\left(\dot{\tilde{w}}_{1 \mathrm{D}}-\dot{w}_{1 \mathrm{D}}\right)+G_{1} \tilde{w}_{1 \mathrm{D}} .
\end{aligned}
$$

For both force- or displacement-controlled conditions this gives a closed ordinary differential equation system with a unique solution.

(a)

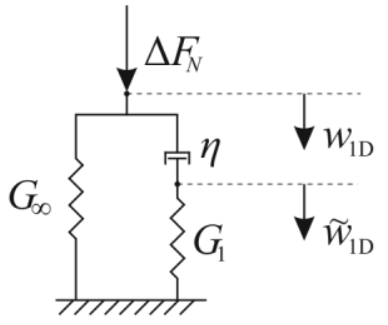

(b)

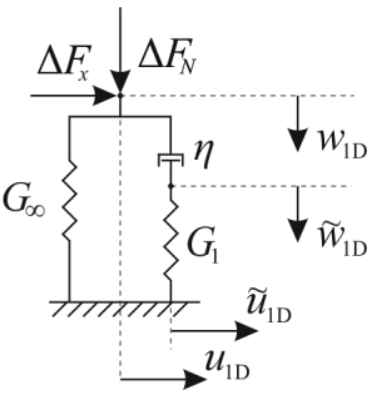

Fig. 2 Rheological standard element of the viscoelastic foundation under normal load (left, a) and superimposed normal and tangential load (right, b)

Note that under force-controlled conditions Eq. (16) can be inverted to give

$$
w_{1 \mathrm{D}}(x, t)=\frac{1}{4 \Delta x} \int_{0}^{t} J\left(t-t^{\prime}\right) \frac{\partial \Delta F_{N}\left(x, t^{\prime}\right)}{\partial t^{\prime}} \mathrm{d} t^{\prime} .
$$

The elements outside the contact area are, of course, free of forces, i.e.

$$
\Delta F_{N}(x, t)=0, \quad|x| \geq a .
$$

An element leaves the contact (dynamically), if the upkeep of contact would require negative normal forces. The total normal force is obtained by summation of all the element normal forces,

$$
F_{N}(t)=\int_{-a(t)}^{a(t)} \frac{\Delta F_{N}(x, t)}{\Delta x} \mathrm{~d} x
$$

and with the linear force density,

$$
q_{z}(x, t):=\frac{\Delta F_{N}(x, t)}{\Delta x},
$$

one can also calculate pressure distribution $p(r, t)$ in the original axisymmetric system by the relation

$$
p(r, t)=-\frac{1}{\pi} \int_{r}^{\infty} \frac{\partial q_{z}(x, t)}{\partial x} \frac{\mathrm{d} x}{\sqrt{x^{2}-r^{2}}} .
$$

We would like to stress again that all the results obtained by the described solution scheme will be exactly correct within the stated assumptions.

Let us now illustrate the procedure by some examples. 


\section{Displacement-controlled indentation}

As the first example we consider the displacement-controlled indentation of a flat three-element standard solid by a rigid cone with slope $\theta$ and thus the profile

$$
f(r)=r \tan \theta .
$$

The indentation depth as a function of time shall be $d(t)=v_{0} t$, which corresponds to a simple indentation test to determine the (visco-)elastic properties of a material. We would like to know the total normal force as a function of time and the material properties of the standard solid.

Solution: The equivalent plain profile is given by

$$
g(x)=\frac{\pi}{2} \tan \theta|x| .
$$

As the contact radius is monotonically increasing Eq. (15) can be applied to determine the contact radius. Hence

$$
a(t)=\frac{2 v_{0} t}{\pi \tan \theta}
$$

An element at position $x$ will therefore come into contact after a time

$$
t_{c}(x)=\frac{\pi}{2} \tan \theta \frac{x}{v_{0}}=\frac{x t}{a(t)} .
$$

The indentation velocity for all the elements in contact is equal $v_{0}$. Hence the application of equation (16) yields the desired normal force:

$$
\begin{aligned}
F_{N}(t) & =8 v_{0} \int_{0}^{a(t)} \int_{x t / a(t)}^{t}\left[G_{\infty}+G_{1} \exp \left(-\frac{t-t^{\prime}}{\tau}\right)\right] \mathrm{d} t^{\prime} \mathrm{d} x \\
& =4 v_{0} a(t)\left[G_{\infty} t+2 G_{1} \tau\left(1+\frac{\tau}{t}\left\{\exp \left(-\frac{t}{\tau}\right)-1\right\}\right)\right] .
\end{aligned}
$$

\section{Force-controlled indentation}

As the second example we consider the force-controlled indentation of a Kelvin-Voigt solid by a rigid sphere with radius $R$. The total normal force shall be kept constant,

$$
F(t)=\text { const }=F_{0} .
$$

This loading situation corresponds to the ideal loading protocol of the commonly used Shore hardness test for elastomers. We would like to know the indentation depth as a function of time and the material properties. 
Solution: The spherical profile can in the vicinity of the contact be approximated by the parabolic profile

$$
f(r)=\frac{r^{2}}{2 R} \Leftrightarrow g(x)=\frac{x^{2}}{R} .
$$

The contact radius will be again, due to creep, a monotonically increasing function with time. Hence

$$
d(t)=\frac{a^{2}(t)}{R} .
$$

In a Kelvin-Voigt solid the stress state is a linear superposition of ideally elastic and ideally viscous stress components (this can be easily understood with the respective rheological model shown in Tab. 1). The total normal force is therefore

$$
\begin{aligned}
F_{N}(t) & \equiv F_{0}=\frac{16}{3} G_{\infty} \frac{a^{3}(t)}{R}+8 \eta \dot{d}(t) a(t) \\
& =\frac{16 G_{\infty}}{3 R}\left[a^{3}(t)+\tau \frac{\mathrm{d}}{\mathrm{d} t}\left(a^{3}(t)\right)\right], \quad \tau:=\frac{\eta}{G_{\infty}} .
\end{aligned}
$$

The solution of this equation with initial condition $a(t=0)=0$ is

$$
a^{3}(t)=\frac{3 F_{0} R}{16 G_{\infty}}\left[1-\exp \left(-\frac{t}{\tau}\right)\right] .
$$

Application of Eq. (31) will then provide the indentation depth as a function of time. The solution in Eq. (33) can be written in the more general form

$$
\frac{F_{N}^{\mathrm{el}}(a(t))}{F_{0}}=\hat{J}(t),
$$

with the elastic (Hertzian) normal force as a function of the contact radius,

$$
F_{N}^{\mathrm{el}}(a):=\frac{16}{3} G_{\infty} \frac{a^{3}}{R},
$$

and the non-dimensional shear creep function for the Kelvin-Voigt solid,

$$
\hat{J}(t):=1-\exp \left(-\frac{t}{\tau}\right) .
$$

As it turns out, the general formulation (34) is correct for arbitrary axisymmetric indenters and arbitrary linear-viscoelastic rheologies ([11], p.232) and can therefore be used to analyse general Shore hardness test configurations. 


\section{Impact test}

As the third example in this section and in order to complete the set of applications of the MDR to commonly used material test of elastomers, we would like to show the application of the described rules to a rebound test: A homogeneous rigid sphere with radius $R$, mass $m$, mass density $\rho$ and initial velocity $v_{0}$ impacts onto a viscoelastic half-space, whose rheology can be described by a three-element standard solid model. We would like to know the coefficient of restitution $e$ (as a measure of energy dissipation under dynamic loading conditions) as a function of the inbound velocity and the material parameters.

Solution: This problem cannot be solved analytically. However, based on the MDR, a simple numerical algorithm can be implemented to give the solution of the impact problem in the quasi-static limit, i.e. assuming that the viscoelastic medium moves through a series of equilibrium states thus neglecting wave propagation in the viscoelastic material. The equation of motion of the sphere, in terms of indentation depth $d$, is simply given by

$$
m \ddot{d}(t)=F_{N}(t)
$$

For all the foundation elements in contact, the displacement is enforced by the movement of the plain parabolic profile equivalent to the three-dimensional sphere (see Eq. (30)),

$$
w_{1 \mathrm{D}}(x, t)=d(t)-\frac{x^{2}}{R}, \quad|x| \leq a .
$$

Solution of Eqs. (18) will give the corresponding element forces. They can be summed up to give the total normal force, which enters the equation of motion. As stated before, an element gets into contact geometrically and leaves contact if contact upkeep would require negative values of the respective element normal force. The impact ends at time $T$, if all elements have left contact. Any time integration scheme can be used to solve the described equation system in discrete time steps, by far the easiest one being an explicit Euler method. As the required computational operations are extremely simple, the time step can be set small enough to ensure numerical stability. The solution of the impact problem, i.e. the coefficient of restitution

$$
e:=-\frac{v(t=T)}{v_{0}}
$$

only depends on two non-dimensional parameters, namely [13]

$$
p_{1}:=\frac{\eta}{R}\left(\frac{v_{0}}{\rho^{2} G_{\infty}^{3}}\right)^{1 / 5}, \quad p_{2}=\frac{G_{1}}{G_{\infty}},
$$

and is shown in Fig. 3 as a function of $p_{1}$ for several different values of $p_{2}$. Note that the physical meaning of $p_{1}$ (except for a numerical factor of the order of unity) is a ratio of two characteristic time scales: the viscoelastic relaxation time of the three-element standard solid compared to the elastic impact duration with $G=G_{\infty}$. 


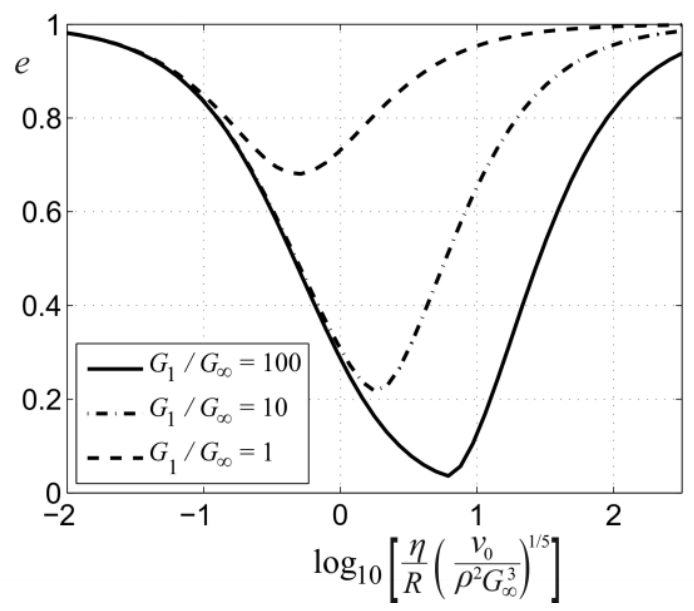

Fig. 3 Coefficient of restitution for the normal impact of a rigid sphere onto

a flat standard solid as a function of the two governing parameters

\section{TANGENTIAL CONTACT OF AXISYMMETRIC BODIES}

We now consider contacts with superimposed normal and tangential loading. Thereby the application of a tangential load is completely analogous to the previous section. The elements of the viscoelastic foundation are vertically and horizontally displaced. Via the superposition principle the tangential force in a single element can be calculated from tangential displacement $u_{1 \mathrm{D}}$ :

$$
\Delta F_{x}(x, t)=\frac{8}{3} \Delta x \int_{0}^{t} G\left(t-t^{\prime}\right) \frac{\partial u_{1 \mathrm{D}}\left(x, t^{\prime}\right)}{\partial t^{\prime}} \mathrm{d} t^{\prime}
$$

Or, vice versa,

$$
u_{1 \mathrm{D}}(x, t)=\frac{3}{8 \Delta x} \int_{0}^{t} J\left(t-t^{\prime}\right) \frac{\partial \Delta F_{x}\left(x, t^{\prime}\right)}{\partial t^{\prime}} \mathrm{d} t^{\prime} .
$$

Alternatively, as in the normal contact problem, the equilibrium conditions for all degrees of freedom of the elements can be evaluated. Coming back to the standard element example in Fig. 2 the equilibrium conditions in tangential direction (see the right side of Fig. 2) read

$$
\begin{aligned}
\Delta F_{x} & =\frac{8}{3} \Delta x\left(G_{\infty} u_{1 \mathrm{D}}+G_{1} \tilde{u}_{\mathrm{ID}}\right), \\
0 & =\eta\left(\dot{\tilde{u}}_{1 \mathrm{D}}-\dot{u}_{1 \mathrm{D}}\right)+G_{1} \tilde{u}_{1 \mathrm{D}} .
\end{aligned}
$$

Note that non-contacting surface areas also relax tangentially. In the frequency domain the convolution (41) reduces to a product,

$$
\Delta \hat{F}_{x}(x, \omega)=\frac{8}{3} \Delta x \hat{G}(\omega) \hat{u}_{1 \mathrm{D}}(x, \omega) .
$$


The tangential linear force density,

$$
q_{x}(x, t):=\frac{\Delta F_{x}(x, t)}{\Delta x}
$$

will provide the total tangential force,

$$
F_{x}(t)=\int_{-a(t)}^{a(t)} q_{x}(x, t) \mathrm{d} x,
$$

and the shear stress distribution in the original axisymmetric system,

$$
\sigma_{x z}(r, t)=-\frac{1}{\pi} \int_{r}^{\infty} \frac{\partial q_{x}(x, t)}{\partial x} \frac{\mathrm{d} x}{\sqrt{x^{2}-r^{2}}} .
$$

To account for micro-slip in the contact we assume the validity of a local AmontonsCoulomb friction law in its simplest form for any contact point: if the local shear stress does not exceed the maximum value given by pressure times friction coefficient $\mu$ the surfaces are able to stick,

$$
\left|\sigma_{x z}(r, t)\right|<\mu p(r, t), \quad \text { stick; }
$$

Otherwise the surface points will slip and the frictional shear stress is known,

$$
\left|\sigma_{x z}(r, t)\right|=\mu p(r, t), \text { slip. }
$$

Thereby it is clear that the contact area will always consist of an inner stick area with radius $c$ and a slip area propagating inside from the edge of contact.

Accounting for slip in viscoelastic frictional contacts within the framework of MDR is simple (and completely analogous to the elastic case): the elements of the viscoelastic foundation simply have to obey the same local Amontons-Coulomb law! That is, if the indenting plain profile is moved tangentially by an increment $\Delta u^{(0)}$ from a given contact configuration, the contacting elements can either stick or slip, defined by the condition

$$
\begin{aligned}
\Delta u_{1 \mathrm{D}}(x, t) & =\Delta u^{(0)}, & & \text { if }\left|\Delta F_{x}(x, t)\right|<\mu \Delta F_{N}(x, t), \\
\Delta F_{x}(x, t) & =\mu \Delta F_{N}(x, t) \operatorname{sgn}\left(u_{1 \mathrm{D}}\right), & & \text { else. }
\end{aligned}
$$

Radius $c$ of the stick area is given by the condition

$$
\left|\Delta F_{x}(c, t)\right|=\mu \Delta F_{N}(c, t) .
$$

\section{Tangential fretting in a viscoelastic contact}

As an illustrative example we consider a simple case of tangential fretting: two axisymmetric bodies with combined relaxation function $G(t)$ are pressed together with a fixed indentation depth $d_{0}$. The equivalent plain profile of non-deformed gap $f(r)$ shall be $g(x)$. After the normal stresses have been relaxed to their asymptotic value, small relative tangential harmonic oscillations

$$
u^{(0)}(t)=\Delta u^{(0)} \cos (\omega t)
$$

are enforced. We would like to know radius $c$ of the permanent stick area. 
Solution: Within the permanent stick area the forces in the elements of the viscoelastic foundation are known to be

$$
\Delta F_{x}(t)=\frac{8}{3} \Delta x \Delta u^{(0)}|\hat{G}(\omega)| \cos (\omega t+\varphi), \quad \varphi=\arg \{\hat{G}(\omega)\} .
$$

Here the complex dynamic modulus, introduced in Eq. (7), has been used because the excitation is harmonic. The normal forces in the fully-relaxed (i.e. elastic) state are

$$
\Delta F_{N}(x)=4 \Delta x G_{\infty}\left[d_{0}-g(x)\right] .
$$

Hence, the radius of the permanent stick area will be given by the solution of the condition

$$
d-g(c)=\frac{2|\hat{G}(\omega)|}{3 \mu G_{\infty}} \Delta u^{(0)}
$$

\section{CONTACT OF COMPRESSIBLE MATERIALS}

Although many (or even most) technically or biologically relevant viscoelastic media can - at least in good approximation - be considered incompressible, any linear isotropic viscoelastic material has not one but two characteristic material functions: in addition to shear relaxation $G(t)$, already used throughout this paper, there is also bulk relaxation function $K(t)$ giving the stress response to a unit volume strain. Accounting for compressibility in viscoelastic contact problems is, in general, a rather non-trivial task [14]. However, for normal contact problems (and only for them) it is easy to show that the compressible contact problem can be traced back to an equivalent incompressible one with the effective shear creep function

$$
J_{\text {eff }}(t)=J(t)+\tilde{J}(t), \quad \tilde{J}^{*}(s)=\frac{3}{s^{2}\left[3 K^{*}(s)+G^{*}(s)\right]} .
$$

This obviously means that in the MDR model the rheological elements of the viscoelastic foundation simply have to be replaced by the elements shown in Fig. 4. For the diagram we assume two materials with relaxation functions $G_{1}(t), G_{2}(t), K_{1}(t)$ and $K_{2}(t)$ respectively. A box, as in the section on rheological models, is an abbreviation for the rheological model representing the relaxation function denoted near the box.

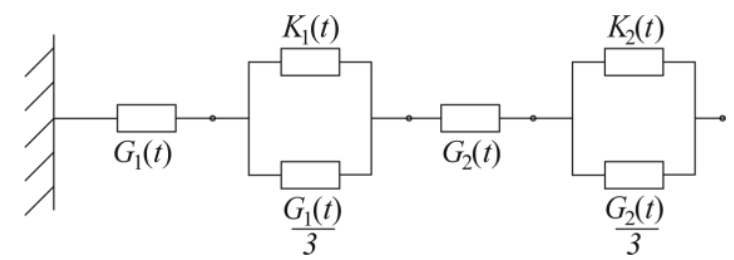

Fig. 4 General rheological element representing two contacting compressible viscoelastic materials. A box is an abbreviation for the rheological model representing the relaxation function denoted near the box 
As compressible media are not by necessity elastically similar to each other we would like to stress that this ascription to an equivalent incompressible problem is only exact for either elastically similar materials or frictionless normal contacts.

\section{Displacement-controlled indentation of a compressible medium}

As an example let us analyse the frictionless indentation of a general Kelvin-Voigt solid with the relaxation functions

$$
\begin{aligned}
& G(t)=G_{\infty}+\eta \delta(t), \\
& K(t)=K_{\infty}+\xi \delta(t),
\end{aligned}
$$

with the shear and bulk viscosities, $\eta$ and $\xi$, by a rigid flat cylindrical punch with radius $a$. The indentation depth as a function of time shall be $d(t)=v_{0} t$. We would like to know the total normal force as a function of time and the material parameters.

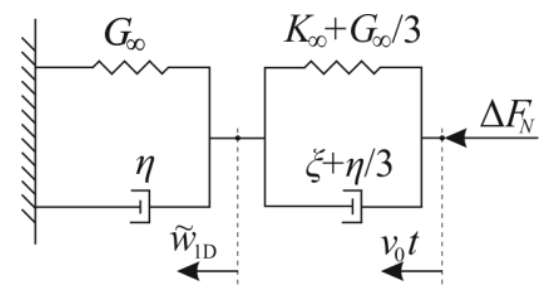

Fig. 5 Displacement-controlled indentation of a compressible Kelvin-Voigt element (based on [8])

Solution: The equivalent MDR-profile of a cylindrical flat stamp is a rectangle of the same length. Hence, all the elements within contact radius $a$ are identically deformed. The equilibrium condition for the inner degree of freedom for each element reads (see Fig. 5)

$$
\left(\xi+\frac{4}{3} \eta\right) \dot{\tilde{w}}_{1 \mathrm{D}}+\left(K_{\infty}+\frac{4}{3} G_{\infty}\right) \tilde{w}_{1 \mathrm{D}}=\left(\xi+\frac{1}{3} \eta\right) v_{0}+\left(K_{\infty}+\frac{4}{3} G_{\infty}\right) v_{0} t .
$$

The solution of this ordinary differential equation with initial condition $\tilde{w}_{1 \mathrm{D}}(t=0)=0$ is given by

$$
\begin{aligned}
\tilde{w}_{1 \mathrm{D}}(t) & =v_{0} \tau(b-c)\left[1-\exp \left(-\frac{t}{\tau}\right)\right]+c v_{0} t, \\
\tau & :=\frac{3 \xi+4 \eta}{3 K_{\infty}+4 G_{\infty}}, b:=\frac{3 \xi+\eta}{3 \xi+4 \eta}, c:=\frac{3 K_{\infty}+G_{\infty}}{3 K_{\infty}+4 G_{\infty}} .
\end{aligned}
$$

Also, for the total normal force (as the indenting body shall be a rigid flat punch, all the elements of the foundation with $|x|<a$ are displaced in the same way),

$$
F_{N}(t)=2 a \frac{\Delta F_{N}(t)}{\Delta x}=8 a\left[G_{\infty} \tilde{w}_{1 \mathrm{D}}+\eta \dot{\tilde{w}}_{1 \mathrm{D}}\right] .
$$


In the limit of fast relaxation $t \gg \tau$ the total normal force will be

$$
F_{N}(t) \approx 8 a v_{0}\left[G_{\infty} \tau(b-c)+G_{\infty} c t+\eta c\right] .
$$

\section{CONCLUSIONS}

The present paper gives a concise description of the rules for the application of the Method of Dimensionality Reduction to contacts of linear viscoelastic materials. Although the given examples mostly focus on analytical solutions for accessibility, it is, of course, possible to implement the rules in a numerical scheme to efficiently simulate viscoelastic contacts with arbitrary oblique loading histories. For example, based on the method, comprehensive contact-impact solutions for viscoelastic materials have been obtained very recently and cross-checked against respective Boundary-Element simulations [13].

\section{REFERENCES}

1. Popov, V.L., Heß, M., 2015, Method of dimensionality reduction in contact mechanics and friction, Springer, Berlin Heidelberg.

2. Argatov, I.I., Heß, M., Pohrt, R., Popov, V.L., 2016, The extension of the method of dimensionality reduction to non-compact and non-axisymmetric contacts, ZAMM Zeitschrift für Angewandte Mathematik und Mechanik, 96(10), pp. 1144-1155.

3. Willert, E., Popov, V.L., 2017, Exact one-dimensional mapping of axially symmetric elastic contacts with superimposed normal and torsional loading, ZAMM Zeitschrift für Angewandte Mathematik und Mechanik, 97(2), pp. 173-182.

4. Heß, M., 2016, A simple method for solving adhesive and non-adhesive axisymmetric contact problems of elastically graded materials, International Journal of Engineering Science, 104, pp. 20-33.

5. Heß, M., Popov, V.L., 2016, Method of Dimensionality Reduction in Contact Mechanics and Friction: A User's Handbook. II. Power-Law Graded Materials, Facta Universitatis-Series Mechanical Engineering, 14(3), pp. 251-268.

6. Argatov, I.I., Popov, V.L., 2016, Rebound indentation problem for a viscoelastic half-space and axisymmetric indenter - Solution by the method of dimensionality reduction, ZAMM Zeitschrift für Angewandte Mathematik und Mechanik, 96(8), pp. 956-967.

7. Kürschner, S., Filippov, A.E., 2012, Normal contact between a rigid surface and a viscous body: Verification of the method of reduction of dimensionality for viscous media, Physical Mesomechanics, 15(5-6), pp. 270-274.

8. Willert, E., Popov, V.L., 2018, Short note: Method of Dimensionality Reduction for compressible viscoelastic media. I. Frictionless normal contact of a Kelvin-Voigt solid, ZAMM Zeitschrift für Angewandte Mathematik und Mechanik, 98(2), pp. 306-311.

9. Popov, V.L., Heß, M., 2014, Method of Dimensionality Reduction in Contact Mechanics and Friction: A Users Handbook. I. Axially-Symmetric Contacts, Facta Universitatis-Series Mechanical Engineering, 12(1), pp. 1-14.

10. Popov, V.L., 2017, Contact Mechanics and Friction. Physical Principles and Applications, 2nd Edition, Springer, Berlin Heidelberg.

11. Popov, V.L., Heß, M., Willert, E., 2018, Handbuch der Kontaktmechanik - Exakte Lösungen axialsymmetrischer Kontaktprobleme, Springer, Berlin Heidelberg.

12. Lee, E.H., Radok, J.R.M., 1960, The Contact Problem for Viscoelastic Bodies, Journal of Applied Mechanics, 27(3), pp. 438-444.

13. Willert, E., Kusche, S., Popov, V.L., 2017, The influence if viscoelasticity on velocity-dependent restitutions in the oblique impact of spheres, Facta Universitatis-Series Mechanical Engineering, 15(2), pp. 269-284.

14. Greenwood, J.A., 2010, Contact between an axisymmetric indenter and a viscoelastic half-space, International Journal of Mechanical Sciences, 52(6), pp. 829-835. 